\title{
AN 8 CM PERIOD ELECTROMAGNETIC WIGGLER MAGNET WITH COILS MADE FROM SHEET COPPER *
}

\author{
G. Biallas" ${ }^{\#}$, S. Benson, T. Hiatt, G. Neil, M. Snyder, Jefferson Lab, Newport News, VA 23606, \\ U.S.A.
}

\section{Abstract}

An electromagnetic wiggler, now lasing at the Jefferson Lab FEL, has 29 eight $\mathrm{cm}$ periods with $\mathrm{K}$ variable from 0.5 to 1.1 and gap of $2.6 \mathrm{~cm}$. The wiggler was made inexpensively in 11 weeks by an industrial machine shop. The conduction cooled coil design uses copper sheet material cut to forms using water jet cutting. The conductor is cut to serpentine shapes and the cooling plates are cut to ladder shape. The sheets are assembled in stacks insulated with polymer film, also cut with water jet. The coil design extends the serpentine conductor design of the Duke OK4 to more and smaller conductors. The wiggler features graded fields in the two poles at each end and trim coils on these poles to eliminate field errors caused by saturation. An added critical feature is mirror plates at the ends with integral trim coils to eliminate three dimensional end field effects and align the entrance and exit orbit with the axis of the wiggler. Details of construction, measurement methods and excellent wiggler performance are presented.

\section{INTRODUCTION}

A conventional machine shop manufactured an electromagnetic (EM) wiggler with $29,8 \mathrm{~cm}$ periods and $\mathrm{K}$ ranging from 0.5 to 1.1 in 11 weeks, for a purchase cost of $\$ 176,000$. The magnet was made entirely using machine shop technology without the use of traditional coils. The wiggler is now lasing, producing light from 1 to $3.1 \mu \mathrm{m}$ in our Upgrade FEL at Jefferson $\mathrm{Lab}^{1,2,3}$. The wiggler is shown in Figure 1

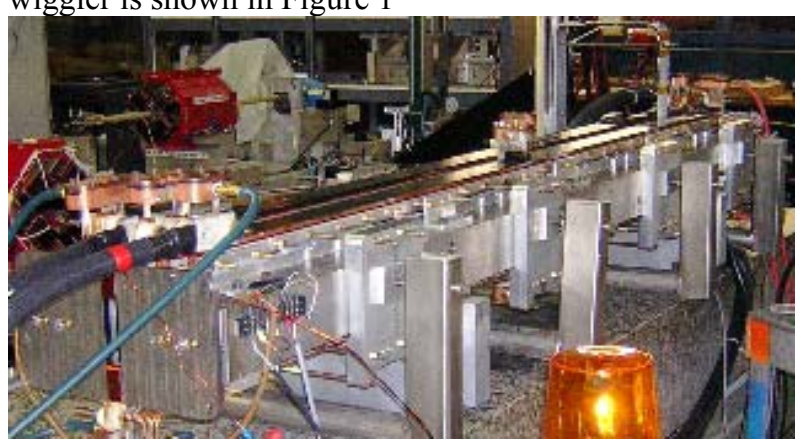

Figure 1 Wiggler on Magnet Test Stand

In response to a critical need for a wiggler with $8 \mathrm{~cm}$ periods, we conceived of an inexpensive and rapid approach to make its coils out of sheet copper. We would make the coils in a serpentine pattern like the undulators used in the Duke OK-4 FEL ${ }^{4}$. The Duke Undulators use 4 internally water-cooled conductors per half, operating at thousands of amperes. We would use many more conduction-cooled conductors made of $1.5 \mathrm{~mm}$ sheet copper to match an existing 220 A power supply. As soon as sheet copper cut to high precision became the basis of our design, we reasoned that water jet cutting would be the fabrication method of choice.

\section{DESIGN}

\section{Four Main Coil Elements}

We set the wiggler gap at $2.6 \mathrm{~cm}$ and fixed on 60 poles, aiming at around 28 periods with $\mathrm{K}=1.0$. We chose to include a safety margin in available amp-turns for saturation effects. We chose 24 turns per pole per half of the wiggler and the resulting measured rms $\mathrm{K}$ of the wiggler vs. current matches with a $2 \mathrm{D}$ analytic model to a few percent. Saturation effects in the actual 3D case were more severe than the model predicted. In addition, the peak field is less due to $3^{\text {rd }}$ harmonic contributions from the shape of the poles. In the end the wiggler achieved $\mathrm{K}$ $=1.1$ with the available power supply.

The water jet cut process allows great design freedom. We extended our water jet cutting based design to make all four of the major coil components, conductor, insulator, cooling plates and clamp plates using this computer controlled rendition of the jigsaw.

The serpentine sheet copper conductors required conduction cooling to a separate, water-cooled cooling plate because a directly attached cooling tube would short out the serpentine turns. With sheet based conductor, we could make the conduction path outside the gaps between poles three times wider than the conductor in the gaps to minimize resistive heating from that part of the conduction path. The conductor could look like a ladder with thin cross bars and thick columns. We chose a current density $6.4 \mathrm{~A} / \mathrm{mm}^{2}$ in the crossbars. A simple alternating slit in the copper columns created a serpentine conduction path. See Figure 2. For cooling, we inserted

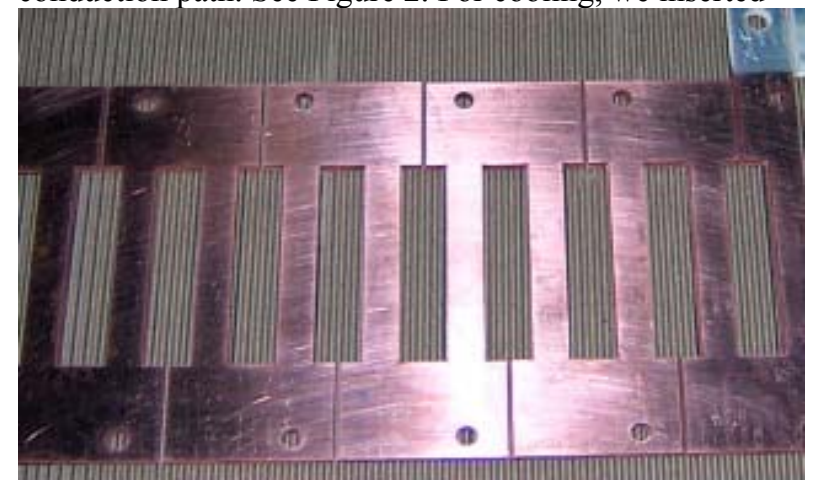

Figure 2 Typical Serpentine Conductor

*Work supported by the US DOE Contract \#DE-AC05-84ER40150, the Office of Naval Research, The Air Force Research Laboratory, the US Army Night Vision Laboratory and the Commonwealth of Virginia, \# Email: biallas@jlab.org 
ladder shaped cooling plates of the same thickness as the conductor between paired conductor sheets. The cooling plates extract the heat by conduction through a layer of insulation, transmitting the heat to the water bearing tubes

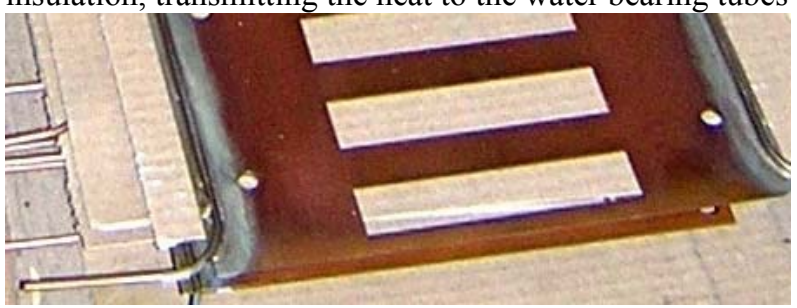

Figure 3 Cooling Plate

brazed to the plate's outer edges. See figure 3. The insulation between conductors and between conductors and cooling plates is $76 \mu \mathrm{m}$ Kapton. We chose it because it has stability at high temperature and is very insensitive to radiation.

The conductor stack, made of 24 conductors, 12 cooling plates and 37 sheets of insulation is held together by the fourth major part, the clamp plates made of $6.3 \mathrm{~mm}$ thick aluminum. Sets of sixty-four $6.3 \mathrm{~mm}$ brass and bronze bolts and studs, thread through the conductor stacks, providing uniformly distributed clamping force. Electrical grade polyester tape insulates the bolts.

\section{End Complexities}

Stepan Mikhailov of the Duke University FEL Center was very helpful in a conversation early in the wiggler design phase. With his advice, we copied the OK4 design's method of grading end pole field's strength to the standard practice of $25 \%$ field integral at the end poles and $75 \%$ at the second poles from the ends. Three sets of conductors end in a connection-loop around the end poles. Six sets of conductors end in connection-loops around the second poles from the end. The remaining three sets of conductors end in a connection-loop around the third poles from the end to provide the grading.

We connect the 24 alternating, serpentine conductors at the head and tail by adding tabs to the ends of the conductors. See Figure 2. The silver-plated tabs stack over one another. Connections at one end are formed when a bolt through the tabs forces tab contact surfaces to make good electrical contact. We insert an insulator film between every other tab-to-tab interface forming sets of doubled conductors. When combined with similar, but staggered-to-alternate-tab connections at the opposite end, a continuous coil is formed. The bolt is insulated from the edges of the tabs by epoxy fiberglass sleeves and washers. The cable lug connections to the wiggler coil are made directly to the tab of a top or bottom serpentine conductor of a stack using the same bolt. This system may be seen in Figure 1.

Per Stepan Mikhailov's suggestions, we added trim coil sets, one set to both end poles and a second set to the second poles in from the ends. We also added mirror plates to the end of the wiggler to cancel out the 3 dimensional end field distortions.

\section{Cores}

We made the new wiggler core's vertical dimension (10 cm thickness) the same as the core of the originally installed Optical Klystron wiggler. A surplus slab of 1006 magnet steel from CEBAF formed the two multi poled core halves, guaranteeing identical magnetic properties in the two halves. The core was annealed using the CEBAF standard vacuum oven, slow cool method for iron cores.

\section{Insulation Details}

Our 40 to 50 volt to ground voltage levels do not challenge the thousands of volts standoff capability of Kapton film. We designed the insulation sheet's form to have a nominal insulation overhang of $1 \mathrm{~mm}$ beyond the edge of any conductor, preventing a short to the grounded cooling plates, clamp plates, poles or bolts. The edges where shorts could happen are found around all 24 conductors at sixty poles, 64 bolt penetrations, and the outer edges to the 12 cooling plates and at two clamp plates. The voltage drop across the magnet is low, minimizing the possibility of flash over along a surface path between conductor and ground. The water jet process achieved tolerances of $\pm 130 \mu \mathrm{m}$ in cutting the film insulation shape. Additional Mylar insulation is applied to the pole tips and the bolts, the objects that penetrate the stack.

\section{Cooling Details}

A compact manifold system distributes the cooling water to the twelve parallel cooling circuits on each side of the conductor stacks. At the ends of the stack, the twelve, $3.2 \mathrm{~mm}$ outside diameter copper water tubes are bent outward in unison. The manifolds are soldered to the tubes after the conductors are compressed into a stack See figure 1. The manifolds are made of a basin with o-ring sealed lid. Soldering the tubes into holes in the bottom of the vessel from its inside permits tests for leak free joints and full flowing tubes before the lid is closed.

\section{MANUFACTURING}

A long standing relationship between Jefferson Lab and an excellent manufacturing company, Process Equipment Co. (PECo) of the Dayton Ohio area allowed them to rapidly establish a fixed price bid on the wiggler. They had the additional advantage of routinely using a very good Dayton water jet company (Kerf Water Jet Co.) and a noted furnace brazing company (Wall Colmonoy). A contract was placed for the Electromagnetic Wiggler on March 8 per a fixed price bid with a promised 10 to 12 week delivery. This period was not our original 10 weeks from lunchtime conversation, but close to it.

We also contracted with PECo for prototype efforts of water jet cutting of both insulation and copper sheets. During this phase we transmitted CAD generated outlines of the parts directly to the water jet vendor. That vendor's software transferred the outlines into cutting code without translation error. We also funded prototype brazing of tubes to cooling plates. The prototyping gave 
PECo and their vendors the confidence to rapidly proceed with production once the production run materials arrived.

The production water jet cutting took about three weeks. Brazing the tubes to the cooling plates took about two weeks. Initial assembly took about a week and a half by several skilled assembly workers and their project manager. However each coil pack had to be disassembled because of low resistance to ground. Small copper chips, probably originating from burrs from the water jet cut edges of the copper were found embedded in and puncturing through the Kapton film in at least four places. The second assemblies took another week and a half and took place in the plant's clean-room-like metrology lab. Despite this precaution, another short to ground appeared in service. We believe a chip is the cause. Fortunately, our power supply could float with respect to ground so we could continue operation of the wiggler. If done again, we would accentuate bur removal and cleaning.

Jefferson Lab received the wiggler 11 weeks after the order was placed.

\section{RESULTS}

The Jefferson Lab semi-automatic hall probe-scanning table measured the wiggler. To allow scanning the probe through the poles, we removed the precision spacer blocks on the upper gap and brought the poles back to parallel using $\mathrm{C}$ clamps. Vertical scans at lower fields show excellent uniformity of the fields in the body of the wiggler. We also found that the field has a $2.5 \%$ third harmonic component that reduces the peak field for a given rms field. The peak field for an rms $\mathrm{K}$ of one is $0.1850 \mathrm{~T}$.

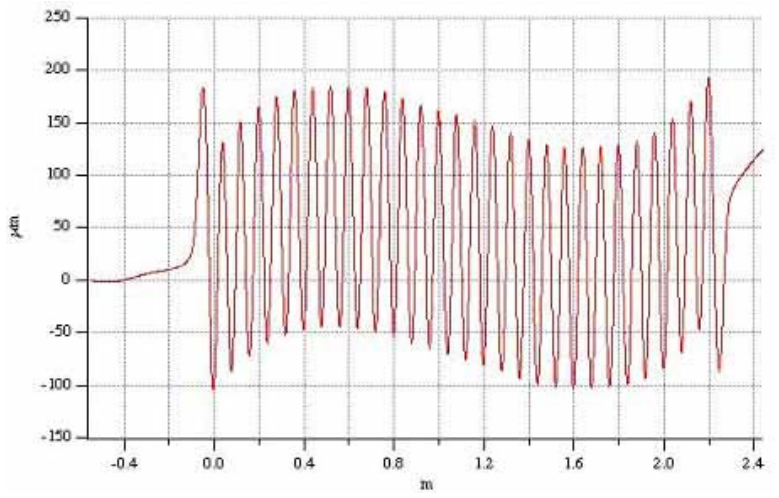

Figure 4, Calculated Trajectory for $\mathrm{K}=1$ Before Using Trim Coils in Series and Adding Coils to Mirror Plates

In early tests, the field strength of the successive body poles increased and decreased in a saw tooth pattern along the length. With this effect, the calculated electron trajectories have a serpentine path through the wiggler. This effect increased greatly at high fields and couldn't be removed by the trim coils while at the same time straightening the entrance and exit trajectories. These results are shown in the first trial at the design rms $\mathrm{K}$ of 1.0 shown in Figure 4.
We found the saw tooth variation in the successive pole fields was caused by saturation in the body core poles that diverted normally symmetrically distributed flux from the third-poles-from-the-ends into the unsaturated $75 \%$ and $25 \%$ strength end poles. By using both sets of corrector coils connected in series in a polarity to buck the excessive flux from the third poles, we were able to straighten the $\mathrm{S}$ shape of the trajectory at all $\mathrm{K}$ values. We added additional windings around the return legs of the mirror plates, forming them into thin window frame corrector dipoles. (See Figure 1.) Powering these coils produced exact straightening of the electron trajectories in and out of the wiggler. Calculated electron trajectories for Ks of 0.65 to 1.05 are shown in Figure 5.

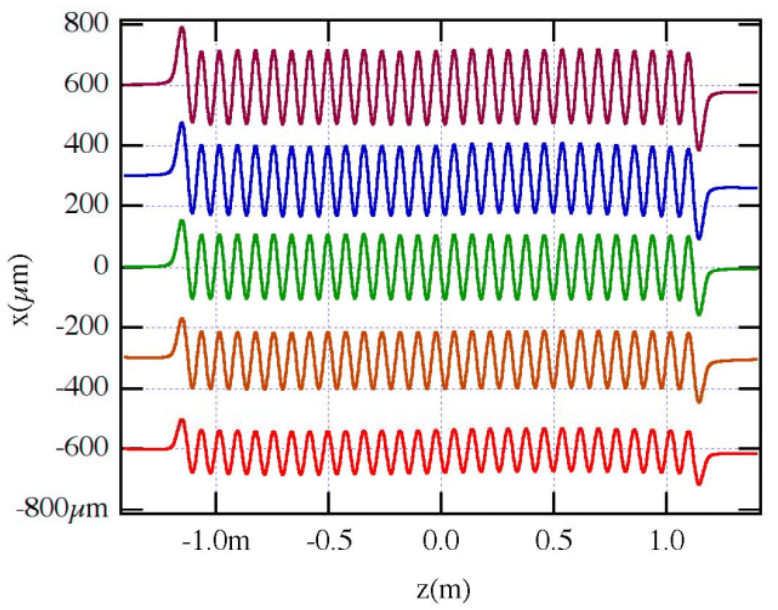

Figure 5 Calculated Trajectories, $\mathrm{K}=0.6535,0.8,0.9$, $1.0, \& 1.05$ from Bottom to Top

\section{CONCLUSIONS}

- Water jet cutting technology is a very cost effective method of making a EM Wiggler at the $8 \mathrm{~cm}$ period and $\mathrm{rms} \mathrm{K}=1$ range.

- Trim coils on the two end poles set to buck out diverted magnetic flux are effective at eliminating the saw tooth field variation caused by saturation.

- $\quad$ Entrance and exit trajectories can be straightened using mirror plates as window frame corrector magnets.

\section{REFERENCES}

[1] D. Douglas et.al. "A $10 \mathrm{~kW}$ IRFEL Design For Jefferson Lab", Proc. PAC2001, Chicago, June 2001.

[2] G. Biallas et.al. "Making an Inexpensive Electromagnetic Wiggler Using Sheet Materials for the Coils", Proc. FEL04, Trieste, Italy, Sept. 2004.

[3] S. Benson et.al., High Power Lasing in the IR Upgrade FEL at Jefferson Lab, Proc. FEL04, Trieste, Italy, September 2004.

[4] G.N. Kulipanov et al, "Thee VEPP-3 Storage-Ring Optical Klystron: Lasing in the visible and ultraviolet Regions", Proc. Of the $11^{\text {th }}$ FEL Conf. Naples, Fl, USA, Aug.-Sept. 1989. 